\title{
PROGRESS TOWARDS A HIGH-RESOLUTION RETINAL PROSTHESIS
}

\author{
James D. Weiland ${ }^{1}$, Wolfgang Fink ${ }^{2,1}$, Mark Humayun ${ }^{1}$, Wentai Liu ${ }^{3}$, Damien C. Rodger ${ }^{4}$, Yu- \\ Chong Tai ${ }^{4}$, Mark Tarbell ${ }^{2}$ \\ ${ }^{1}$ Doheny Retina Institute, Doheny Eye Institute, Department of Ophthalmology, Keck School of Medicine, \\ University of Southern California, Los Angeles, CA \\ ${ }^{2}$ Division of Physics, Mathematics and Astronomy, California Institute of Technology, Pasadena, CA, USA \\ ${ }^{3}$ Department of Electrical Engineering, University of California, Santa Cruz, CA, USA \\ ${ }^{4}$ Bioengineering, Division of Engineering and Applied Science, California Institute of Technology, \\ Pasadena, CA, USA
}

\begin{abstract}
Simulations of artificial vision suggest that 1000 electrodes may be required to restore vision to individuals with diseases of the outer retina. In order to achieve such an implant, new technology is needed, since the state-of-the-art implantable neural stimulator has at most 22 contacts with neural tissue. Considerable progress has been made towards that goal with the development of image processing, microelectronics, and polymer based electrodes and interconnects. An image processing system has been realized that is capable of real-time implementation of image decimation and filtering (for example, edge detection). Application specific integrated circuits (ASICs) have been designed and tested to demonstrate closed loop power control and efficient microstimulation. A novel packaging process has been developed that is capable of simultaneously forming a receiver coil, interconnects, and stimulating electrodes.
\end{abstract}

Keywords - retinal prosthesis, microsystems, electrical stimulation, neural prostheses

\section{INTRODUCTION}

Several incurable eye diseases result in blindness for 100,000's of individuals each year.[1] One proposed treatment for these conditions is a retinal prosthesis that will stimulate the retina at many distinct locations to create a pattern of neural activation and thus a visual perception.[2] In order to function properly, a retinal prosthesis will require the presence of cells in the retina. Therefore, diseases that are primarily limited to the outer retina are potentially treatable with a retinal prosthesis. The two most common outer retinal degenerative diseases are age-related macular degeneration (AMD) and retinitis pigmentosa (RP). AMD is more prevalent but RP is more severe. Electrical stimulation in human test subjects with these conditions, has demonstrated the feasibility of an electronic retinal prosthesis as a means of providing some degree of vision.[3] This paper describes progress towards a high-resolution retinal prosthesis designed to restore visual functions such as face recognition, mobility, and navigation.

\section{BACKGROUND}

In order to design a high resolution retinal prosthesis, it is important to consider the system requirements with respect to the diseased tissue, the number of pixels needed for the patient to have useful vision, and the output requirements to elicit perception of light with an electrode on the retinal surface.

Post-mortem evaluation of retina with RP or AMD has shown a large number cells remain in the inner retina compared to the outer retina. In severe RP, in the macula only $4 \%$ of photoreceptors remained but $80 \%$ of inner retina and $30 \%$ of ganglion cells remained.[4, 5] In extramacular regions, only $40 \%$ of inner retina remained. In AMD, $90 \%$ of the inner retina cells remained compared to age-matched controls.[6] Thus, by measures of cell counting, the inner retina in RP and AMD appears to be less affected by disease compared to the photoreceptors. Further, electrical stimulation of humans with RP and AMD results in the perception of light, so some neural elements exist that can be activated. Recent studies using novel cell tracing, however, suggest that the inner retina undergoes significant remodeling during retinal degeneration.[7] Further, Mueller cell hypertrophy leads to a seal between the subretinal space and the inner retina. In summary, RP and AMD do not result in complete degeneration of the retina, but it may not be correct to assume that the information processing circuitry of the inner retina is intact.

Simulations of prosthetic vision with a retinal implant have predicted the number of electrodes needed to perform certain visual tasks. These simulations activated a 17 degree area of the retina, which is similar to the coverage of some prototype retinal prostheses. Dagnelie, et al., have studied pixelized vision using a modified Low Vision Enhancement System (LVES) to pixelize vision and stabilize the image on the retina (image stabilization is done through eye tracking).[8] Normally-sighted subjects used pixelized vision to recognize faces at rates that were significantly above chance with as few as $10 \times 10$ pixels ( $60 \%$ correct versus $25 \%$ chance). When $32 \times 32$ electrodes were used, the recognition scores improved to over $80 \%$. Pixel dropout of $70 \%$ led to worse scores that were equivalent to guessing (dropout means the pixel is turned off, and is analogous to a non-functional electrode). Using a similar system, Hayes, et al., studied performance in a number of different visual tasks including reading.[9] Reading speeds of 15 words per minute were possible, even with only a $16 \times 16$ pixelized view. While this is below 
normal reading speed, it does approach a level of utility that may be acceptable to a blind individual. Thus a conservative estimate of pixel density would be 1000 pixels in the macula ( $5 \mathrm{~mm}$ diameter area of central retina) to restore this level of vision with a retinal prosthesis.

A recent clinical trial of a chronically implanted prototype device has demonstrated perception thresholds can be less than $50 \mathrm{nC}$, if the stimulus electrode is positioned in close proximity to the retina.[10] Thresholds equal to or less than this level were obtained on 14 of 16 electrodes in 2 subjects, while in a third subject higher thresholds were found. While a larger data set will be needed to make statistically meaningful correlations, the third subject had suffered from a longer period of retinal degeneration (over 50 years) which may be partially responsible for the discrepancy. This was a significant result since prior clinical trials with temporarily implanted devices had shown much higher thresholds, which would have called into question the safety of a chronic implant. Thus an electrode capable of providing $50 \mathrm{nC}$ of charge safely will be needed.

\section{RESULTS}

The goal of our research group is to develop a high-resolution retinal implant that can restore vision so that blind individuals can read, recognize faces, and navigate in unfamiliar environments. In order to achieve this goal, we envision a system with external image acquisition and processing, an implanted microstimulator with 1000 contacts to the retina, and a wireless telemetry system linking the external and implanted components. The results below detail our progress in these areas.

Image processing will provide the transformation from image to stimulus pattern. The retina performs a significant amount of image processing between the photoreceptors (image detection) and retinal ganglion cells (retinal output). Since our device will stimulate at the retinal ganglion cell layer, it may be necessary to replicate retinal processing to recreate a natural retinal output. Alternatively, the brain may learn to adapt to the stimulation input provided by our device and therefore computationally less demanding filters may be adequate. Given the uncertain condition of the diseased retina (described above), our initial image processing system will be flexible and will have significant computational capability. We have created a first generation image processing system.[11] A firewire (IEEE 1394) camera streams video to a laptop computer. The software package comprises numerous efficient image manipulation and processing algorithms/modules that have been developed to modify the captured video stream in real-time (30 fps) such as (see Figure 1):

- Video pixelization to simulate the vision provided by a retinal implant electrode array

- Contrast and brightness correction/enhancement
- Grayscale histogram equalization for luminance control under severe contrast and brightness conditions

- Reduction of available (user-defined) grayscale levels

- Edge detection.

- User-definable nearest-neighbor-based blurring algorithms may enhance significantly the otherwise low-resolution pixelated image provided by a retinal implant

- Implementation of capability to allow for repeated application of image manipulation and processing modules in arbitrary, user-defined order.

While a laptop is potentially a portable system, we are beginning to implement these algorithms on a Digital Signal Processor (DSP). The final system may use a laptop as a fitting system for each patient and a DSP based unit to run the optimized and personalized set of filtering algorithms.
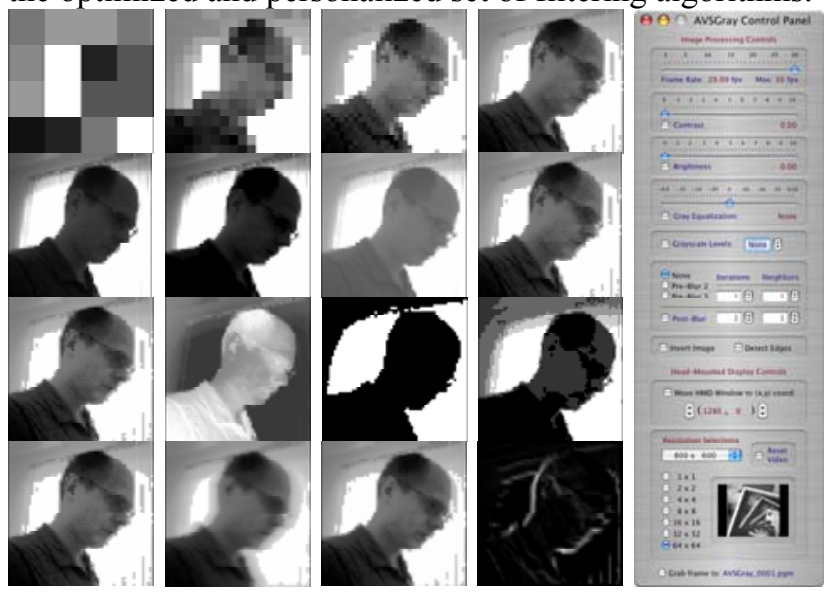

Fig. 1 - Image Processing for an Intraocular Retinal Prosthesis. This system runs in real-time on a laptop computer and performs image decimation (top row), contrast and brightness equalization (second row), inversion and custom grayscale (third row), and blurring and edge enhancement (bottom row).

Microelectronics components are under development to support the requirements of 1000 electrodes and $50 \mathrm{nC}$ output per electrode. The telemetry system will provide power and data to the implanted unit. One fundamental challenge in designing telemetry systems for biomedical implants is the competing requirements between transmission of power (better at low frequency) and data (higher data rates need high frequency). Since the data rate for a 1000 channel retinal prosthesis may be as high as 2 Mbps, we separated the power and data into separate frequency bands (power $1 \mathrm{MHz}$, data $16 \mathrm{MHz}$ ). The power transmitter and receiver have been realized in the form of custom integrated circuits and discrete components. Unlike conventional methods of open loop power transmission we have developed an adaptive scheme that senses the change in the power requirements in the implant and suitably adjusts the transmitted power automatically (see Figure 2). This closed loop control also counteracts any changes in 
the coil coupling and reduces additional power dissipation. For microstimulation of neural tissue, a prototype analog driver has been fabricated.[12] The driver circuitry is capable of reducing the stimulus current without compromising the resolution. This will allow reduction in stimulus current after the implantation thus reducing power consumption.

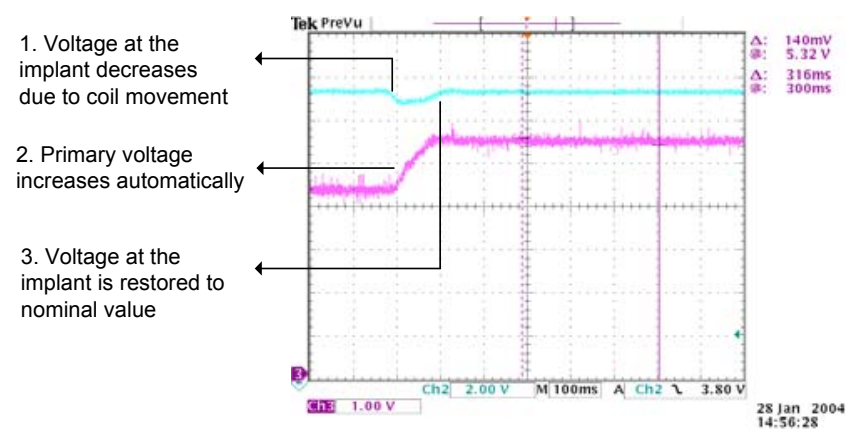

Fig. 2 - Closed Loop Power control. The transmitted power is automatically adjusted to maintain the implant power supply.

Connecting the various elements of the implanted systems will be done with a novel technology capable of achieving the lead density of 1000 conductors between the stimulator chip and the electrode array. This approach, now known as the chip-level integrated interconnect $\left(\mathrm{CL}^{2}{ }^{2}\right)$ technology, can simultaneously achieve coil, interconnects, and electrodes in a single process. In this process, an ASIC is placed and sealed into a precisely located hole in a silicon wafer. Microfabrication on top of this chip, with precise alignment, can be carried out as though this chip's circuitry had been initially fabricated in the host substrate itself. A sacrificial layer of photoresist is spun on the wafer. Parylene $\mathrm{C}$ is then deposited, and contact holes to the underlying chip are etched in this parylene $\mathrm{C}$ (using standard photolithography). Metal traces and electrodes are patterned on top of this parylene (using standard photolithography), with optimized step coverage for achieving electrical contact to the underlying chip. A second parylene layer is deposited to seal this circuitry. Electrodes are then exposed by oxygen plasma etching, and the entire structure is lifted off from the underlying substrate through removal of the photoresist sacrificial layer (see Figure 3). $6 \mu \mathrm{m}$ lines and spaces have been demonstrated, so using a multi-layer metal process, it is feasible to incorporate 1000 lines into the $5 \times 5 \mathrm{~mm}^{2}$ space requirement. Using a test chip with conducting lines, the process has demonstrated excellent connectivity.

\section{CONCLUSION}

Several important technology advances have been made towards a high resolution retinal prosthesis. Remaining challenges include circuit design of a 1000 channel stimulator and hermetic packaging.

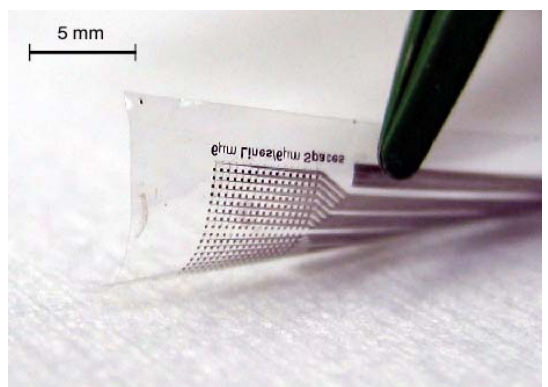

Fig. 3 - Parylene electrode array with 256 contacts in a 5x5 mm space, demonstrating a high density capability for this technology.

\section{ACKNOWLEDGMENT}

This material is based on work supported by the National Science Foundation under Grant No. EEC-0310723.

\section{REFERENCES}

1. Sharma, R.K. and B. Ehinger, Management of hereditary retinal degenerations: present status and future directions. Surv.Ophthalmol, 1999. 43(5): p. 427.

2. Margalit, E., et al., Retinal prosthesis for the blind. Surv.Ophthalmol., 2002. 47(4): p. 335.

3. Humayun, M.S., et al., Visual perception in a blind subject with a chronic microelectronic retinal prosthesis. Vision Research, 2003. 43(24): p. 2573.

4. Stone, J.L., et al., Morphometric analysis of macular photoreceptors and ganglion cells in retinas with retinitis pigmentosa. Arch Ophthalmol, 1992. 110(11): p. 1634

5. Santos, A., et al., Preservation of the inner retina in retinitis pigmentosa. A morphometric analysis. Arch Ophthalmol, 1997. 115(4): p. 511.

6. Kim, S.Y., et al., Morphometric analysis of the macula in eyes with disciform age-related macular degeneration. Submitted to Arch Ophthalmol, 2001.

7. Marc, R.E., et al., Neural remodeling in retinal degeneration. Prog Retin Eye Res, 2003. 22(5): p. 607.

8. Thompson, R.W., et al., Facial recognition using simulated prosthetic pixelized vision. Invest Ophthalmol Vis Sci, 2003. 44(11): p. 5035.

9. Humayun, M.S., et al., Visual perception elicited by electrical stimulation of retina in blind humans. Arch Ophthalmol, 1996. 114(1): p. 40.

10. Mahadevappa, M., et al., Electrical Stimulus Parameters for Visual Perception in Blind Humans with Retinal Prosthetic Implants. Invest Ophthalmol Vis Sci, 2003. 43: p. 5059.

11. Liu, W., et al. Image Processing and Interface for Retinal Visual Prostheses. in ISCAS. 2005. Kobe, Japan.

12. Sivaprakasam, M., et al., A Variable Range BiPhasic Current Stimulus Driver Circuitry for an Implantable Retinal Prosthetic Device. Journal of Solid State Circuits, 2005. 40(3): p. 763-771. 